\title{
Detailed Calculation of Test-Mass Charging in the LISA Mission
}

\author{
H. M. Araújo, P. Wass, D. Shaul, G. Rochester and T. J. Sumner \\ Blackett Laboratory, Imperial College London, SW7 2BW, UK
}

\begin{abstract}
The electrostatic charging of the LISA test masses due to exposure of the spacecraft to energetic particles in the space environment has implications in the design and operation of the gravitational inertial sensors and can affect the quality of the science data. Robust predictions of charging rates and associated stochastic fluctuations are therefore required for the exposure scenarios expected throughout the mission. We report on detailed charging simulations with the Geant4 toolkit, using comprehensive geometry and physics models, for Galactic cosmic-ray protons and helium nuclei. These predict positive charging rates of $50 \mathrm{He} / \mathrm{s}$ (elementary charges per second) for solar minimum conditions, decreasing by half at solar maximum, and current fluctuations of up to $30 \mathrm{+e} / \mathrm{s} / \mathrm{Hz}^{1 / 2}$. Charging from sporadic solar events involving energetic protons was also investigated. Using an event-size distribution model, we conclude that their impact on the LISA science data is manageable. Several physical processes hitherto unexplored as potential charging mechanisms have also been assessed. Significantly, the kinetic emission of very low-energy secondary electrons due to bombardment of the inertial sensors by primary cosmic rays and their secondaries can produce charging currents comparable with the Monte Carlo rates.
\end{abstract}

Key words: LISA, space environment

PACS: 95.55.Ym, 04.80.Nn, 07.05.Tp

\section{Introduction}

LISA, the Laser Interferometer Space Antenna, is a joint ESA/NASA mission designed to detect gravitational waves by means of space-borne interferometric measurements. Gravitational disturbances will induce minute variations in the relative separation of free-floating test masses (TMs), which are distributed in pairs in an equilateral constellation of three spacecraft 5 million $\mathrm{km}$ apart [1]. 
The radiation environment which will be encountered by LISA in its Earthlike orbit around the Sun can threaten its ambitious target sensitivity for detecting gravitational waves. The precursor mission LISA Pathfinder (formerly known as SMART-2) will be similarly vulnerable at the Earth-Sun L1 Lagrange point [2]. Spurious TM accelerations result from the accumulation of charge on the isolated masses caused by the bombardment of the spacecraft by cosmic rays and solar particles. A charged TM interacts electromagnetically with surrounding conducting surfaces as well as with magnetic fields in interplanetary space. These spurious Coulomb and Lorentz forces lead to both coherent signals and in-band noise which can limit the sensitivity to gravitational waves.

TM charging is a consideration in the design of the inertial sensor (electromagnetic analysis, materials, shielding). Subsequently, it drives the development of an effective charge management system (charge measurement, neutralisation, operation modes, recovery from drastic charging events). The unavoidable contamination of the science data needs to be assessed and mitigated using data analysis techniques, possibly assisted by information from diagnostic tools such as particle monitors.

Previous work has established approximate charging rates from Galactic cosmic rays at solar minimum conditions and the magnitude of their spurious acceleration signals $[3,4,5,6,7,8]$. These studies were based on Monte Carlo (MC) simulations using the Geant3 [9] and Geant4 [10] toolkits. Simulation work with the Fluka package [11] is also ongoing [12]. Positive charging rates in the range $11-29+\mathrm{e} / \mathrm{s}$ result from these studies, ${ }^{1}$ depending on the complexity of the models and TM size considered. In this work we set out to improve on previous calculations for LISA by using more sophisticated physics models, a detailed implementation of the spacecraft geometry, and by assessing other possible charging mechanisms not considered previously. A similar study for LISA Pathfinder is described elsewhere [13].

$\overline{1}$ Note: a normalisation error was made in Ref. [6] in scaling the MC to the omnidirectional GCR flux. Consequently, charging rate estimates found there are inflated by a factor of 2 . This has been accounted for in the comparisons presented in this article. 


\section{Geant4 Simulation}

\subsection{Geometry Model}

The Geant4 implementation of the LISA Science Module, illustrated in Fig. 1, was based on the LISA Integrated Solid Model [14]. Some 200 volumes represent nearly all components above $\sim 0.1 \mathrm{~kg}$ at the correct location, using the assigned materials when known. Overall, almost $85 \%$ of a total spacecraft mass of $400 \mathrm{~kg}$ has been accounted for; numerous small pieces of structural hardware make up most of the missing mass.

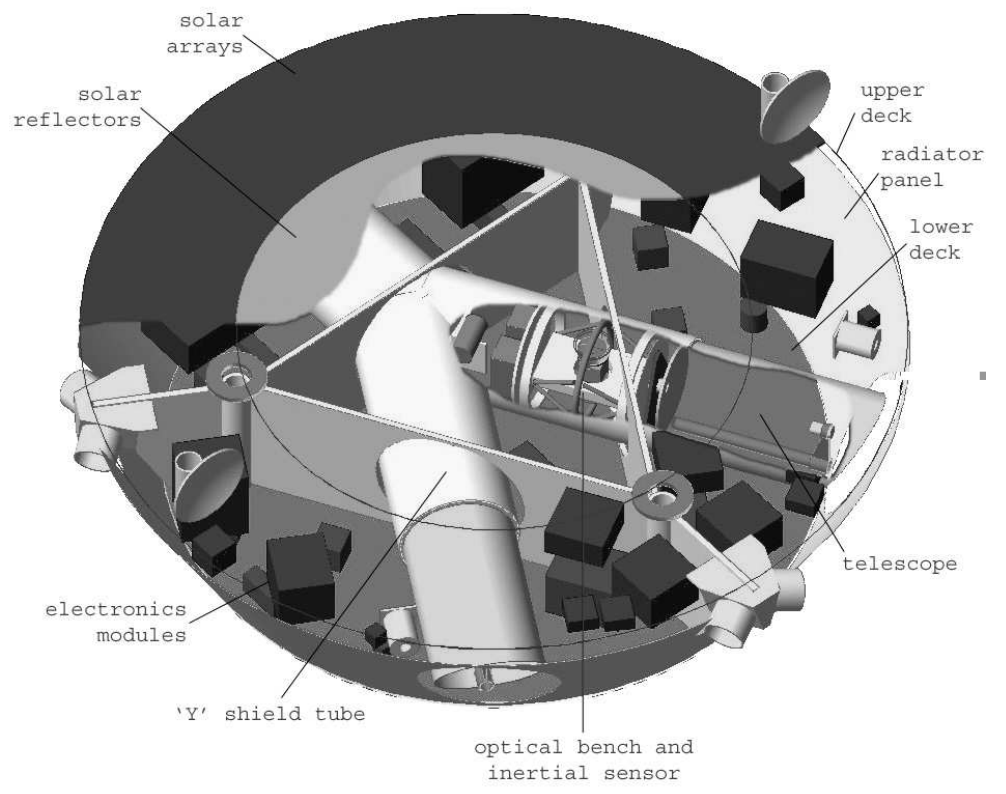

Fig. 1. LISA spacecraft model implemented in Geant4. Two inertial sensors are located inside the Y-shaped payload structure (one is shown in the figure).

Each LISA module accommodates two interferometer telescopes mounted inside a Y-shaped payload structure. The outer spacecraft structure is approximately $2.7 \mathrm{~m}$ in diameter and $0.5 \mathrm{~m}$ in height. Two TMs are housed in inertial sensors (IS) located in optical benches mounted behind the telescopes. The IS representation is based on the current design of the LISA Technology Package (LTP) sensor [15] aboard the LISA Pathfinder. An important difference between the earlier LISA and the new LTP sensor designs is a larger TM considered in the latter (46 mm cube, as opposed to $40 \mathrm{~mm}$ ). The Geant 4 model is represented in a cutaway view in Fig. 2. The test cube is modelled 
as $\mathrm{Au}_{.7} \mathrm{Pt}_{.3}$ alloy and is surrounded by sensing and actuation electrodes $(Y-Z$ injection configuration) lodged in a molybdenum housing. A $0.3 \mu \mathrm{m}$ gold layer plates the entire inner surface of the sensor housing. Caging mechanisms, used to immobilise the cube during launch, are shown in Fig. 2 above and below the sensor. The assembly is accommodated in a titanium vacuum enclosure, the edges of which are also shown.

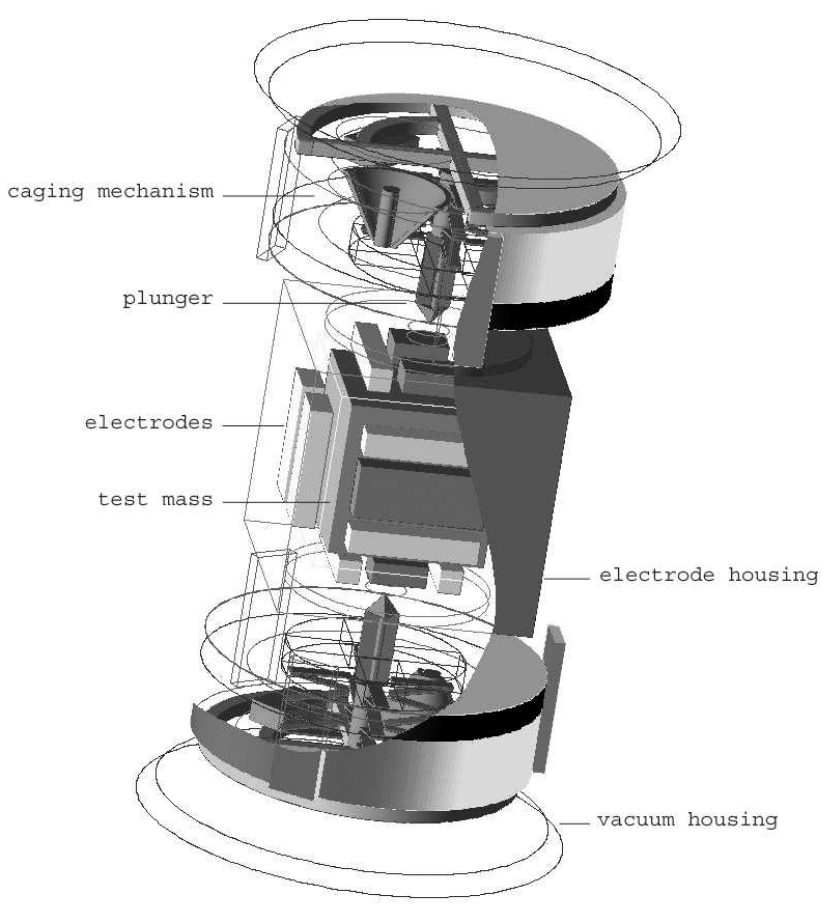

Fig. 2. LTP inertial sensor implemented in Geant4. The TM, located at the centre of the figure, is surrounded by sensing electrodes (light grey) and injection electrodes (dark grey). Caging mechanisms can be seen above and below the electrode housing.

\subsection{Simulation of the Radiation Environment}

In the science phase of the LISA mission, two particle fluxes will dominate the charging rates occurring in its test masses: a permanent background flux of Galactic cosmic-ray (GCR) protons and light nuclei and, occasionally, solar energetic particles (SEP) driven by shock acceleration from certain types of solar event.

The GCR spectrum varies in intensity during the 11-year long solar cycle. The interplanetary magnetic field is weakest at the minimum of solar activity; as a consequence, a higher GCR flux is expected in the solar cavity. At solar quiet, approximately $90 \%$ of the particle flux consists of protons, $8 \%$ of $\mathrm{He}$ 
nuclei $\left({ }^{3} \mathrm{He}\right.$ and $\left.{ }^{4} \mathrm{He}\right), 1 \%$ of heavier nuclei and $1 \%$ of electrons. Conversely, the lowest GCR fluxes are expected at solar maximum. The three most abundant primary nuclei (p, $\left.{ }^{3} \mathrm{He},{ }^{4} \mathrm{He}\right)$ at solar minimum and maximum fluxes were the main simulation inputs. The TM charging from SEP events was determined separately, and is discussed in Section 4. The effect of heavier nuclei is being examined, but it is not thought to be a significant one. Work is under way to address the issue of GCR variability in the LISA bandwidth and flux directionality. In the present work GCR fluxes are considered constant and isotropic, which should not affect the main conclusions reached.

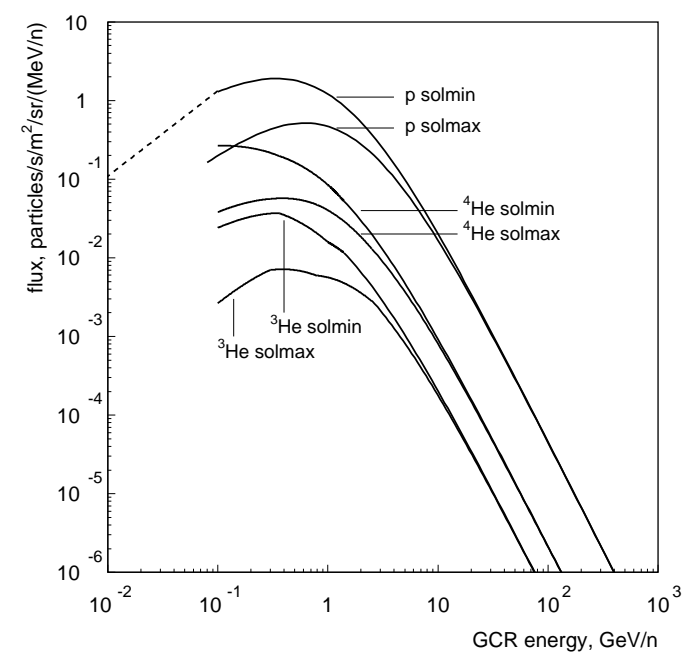

Fig. 3. Differential energy spectra for GCR protons and He nuclei at 1 AU.

The adopted GCR spectra are those shown in Fig. 3 [17]. Since most spectral data do not distinguish between the two He isotopes, a simple parameterisation of the ratio ${ }^{3} \mathrm{He} /{ }^{4} \mathrm{He}$ was used [18]. Only energies above $100 \mathrm{MeV} / \mathrm{n}$ were considered for the simulation input, the minimum required to penetrate the shielding overlying the TMs, except for the solar minimum proton spectrum, which was extended down to $10 \mathrm{MeV} / \mathrm{n}$ in order to establish this fact.

In carrying out the $\mathrm{MC}$ simulation, the isotropic primary flux is generated from points sampled uniformly from a spherical surface encompassing the whole geometry, biased with a cosine-law angular distribution around the surface normal. The particle energy is sampled randomly from the GCR spectra. The MC timelines are normalised by considering that $N_{0}$ primaries represent a fluence $\Phi=N_{0} /\left(\pi R^{2}\right)$ particles per unit area anywhere inside a generator surface of radius $R$. 


\subsection{Physics Models}

Owing to their high energy and hadronic nature, GCR interactions entail complex nuclear reactions which have large final-state multiplicities, producing a plethora of secondaries. All these particles need to be tracked down to the lowest possible energy, especially those approaching the IS. As a result, many of the physics models available in Geant 4 are required for this simulation; they describe hadronic, electromagnetic and photonuclear interactions, spanning almost 10 orders of magnitude in energy. A detailed description of these models can be found in Ref. [16].

A milestone in the application of Geant4 to cosmic-ray simulations was the implementation of intra-nuclear cascade models for the inelastic scattering of nucleons, followed by their recent extension to light nuclei. For protons, these are complemented by a quark-gluon string model above $\sim 6 \mathrm{GeV}$. Nuclear evaporation models treat the excited residual nucleus as well as primaries below $\sim 100 \mathrm{MeV}$. The new models improve the treatment of GCR protons and allow, for the first time, a realistic simulation of He fluxes.

A threshold of $250 \mathrm{eV}$ was imposed for secondary particle production inside the IS, with the exception of delta rays (knock-on electrons) directly created by hadron ionisation; these are currently suppressed below the mean excitation energy of the material $(\sim 800 \mathrm{eV}$ in gold). The effect of these production cuts is analysed later. Another important issue in this simulation is the backscattering of electrons at material boundaries. Backscattering fractions for heavy elements such as gold and platinum can be as large as 50\% above $\mathrm{keV}$ energies. The Geant4 model was tuned to reproduce the fraction, energy and angular distributions of backscattered electrons found experimentally for these materials.

The charging potential of several additional physics processes was assessed separately from the simulation. Mechanisms such as the kinetic emission of very low energy electrons and atoms (sputtering) are not modelled by Geant4. Others, such as transition radiation and Cherenkov emission, could have been included in the $\mathrm{MC}$, but we opted for a separate treatment due to their intricate dependence on the detailed IS design. A prototype model of protoninduced x-ray emission does exist in Geant4, but it proved too inaccurate for our purpose. Finally, mainly for a speedier simulation, the effects of cosmogenic activation and radioactive decay of the spacecraft materials were investigated independently. All these processes are discussed in Section 5. 


\section{Simulation Results}

The MC model tracks cosmic rays and secondary particles capable of penetrating the IS shielding (and all secondaries produced inside it), tallying those entering and leaving the two test masses $\left(\mathrm{TM}_{0}\right.$ and $\left.\mathrm{TM}_{1}\right)$. Generation of the $\mathrm{MC}$ data required $\sim 1 \mathrm{CPU}$ year to achieve convergence of the charging rates to within a few percent [19]. A minimum exposure time $T=400 \mathrm{~s}$ was simulated for both solar minimum and solar maximum conditions, totalling $N_{0}=2.2 \times 10^{8}$ simulated events, distributed as shown in Table 1 . The omnidirectional GCR fluxes above $100 \mathrm{MeV}, F$, are also indicated. On average, one charging event was registered for $\sim 1000$ primaries striking the spacecraft. Some events were found to deposit charge on both masses simultaneously, but their number was negligible.

\subsection{Mean Charging Rates and Fluctuations}

The average charging rate of each TM, $R \pm \sigma_{M}$, and the spectral density $S_{R}$ of the shot noise associated with the charging current are summarised in Table 1, for a constant and isotropic GCR flux. The MC uncertainty, $\sigma_{M}$, is calculated by combining the Poisson variances for the occurrence of each net event charge. All contributions to the total charging rate are positive for both solar conditions, and slightly different for the two masses (but almost within errors). A small discrepancy is to be expected: although the IS occupy almost symmetrical positions in the spacecraft, they were placed with the same orientation inside the payload arms and not as mirror images with respect to the axis of symmetry. As a result, the masses see slightly different amounts of shielding. For $\mathrm{TM}_{1}$, located in the IS visible in Fig. 1, the rates are $23.7 \pm 0.6+\mathrm{e} / \mathrm{s}$ and $49.9 \pm 0.9+\mathrm{e} / \mathrm{s}$ for solar maximum and solar minimum fluxes, respectively. The solar minimum value is almost twice the corrected figure obtained from Ref. [6] $(29+\mathrm{e} / \mathrm{s})$. Although the proton flux clearly dominates these rates, it is worth noting that He, which constitutes only $8 \%$ of the total GCR flux, contributes about twice that fraction to the total charging rate.

To highlight the stochastic nature of the charging process, we write the timedependent fluctuations of the total charge as $\delta Q(t)=Q(t)-R t$, shown in Fig. 4. Besides adding to the acceleration noise, these fluctuations also set the ultimate accuracy expected from a finite measurement of the total TM charge.

For a more rigorous analysis, we write $R$ in terms of statistically independent contributions from events giving only a net charge $q e(q=+1$ for protons). The parallel can then be made with the transport of equal charges to and from a charge reservoir, i.e. electrical shot noise. The distribution of individual 
Table 1. Summary of the Monte Carlo Results

\begin{tabular}{|c|c|c|c|c|c|c|c|c|c|c|}
\hline \multirow{2}{*}{$\begin{array}{l}\text { primary } \\
\text { particle }\end{array}$} & \multirow{2}{*}{$\begin{array}{c}\text { solar } \\
\text { activity }\end{array}$} & \multirow{2}{*}{$\begin{array}{l}\text { GCR flux } \\
F, / \mathrm{s} / \mathrm{cm}^{2}\end{array}$} & \multicolumn{2}{|c|}{ timeline } & \multicolumn{3}{|c|}{$\mathrm{TM}_{0}$} & \multicolumn{3}{|c|}{$\mathrm{TM}_{1}$} \\
\hline & & & $N_{0}, 10^{8}$ & $T, \mathrm{~s}$ & $R,+\mathrm{e} / \mathrm{s}$ & $\sigma_{M}, \mathrm{e} / \mathrm{s}$ & $S_{R}, \mathrm{e} / \mathrm{s} / \mathrm{Hz}^{1 / 2}$ & $R,+\mathrm{e} / \mathrm{s}$ & $\sigma_{M}, \mathrm{e} / \mathrm{s}$ & $S_{R}, \mathrm{e} / \mathrm{s} / \mathrm{Hz}^{1 / 2}$ \\
\hline $\mathrm{p}$ & & 4.29 & 121.1 & 400 & 39.8 & 0.8 & 21.2 & 41.2 & 0.8 & 21.5 \\
\hline${ }^{4} \mathrm{He}$ & $\min$ & 0.315 & 14.4 & 642 & 7.2 & 0.3 & 10.5 & 7.7 & 0.3 & 11.0 \\
\hline${ }^{3} \mathrm{He}$ & & 0.0591 & 14.1 & 3366 & 1.08 & 0.05 & 3.95 & 1.04 & 0.05 & 4.05 \\
\hline total & & 4.66 & 149.6 & - & 48.1 & 0.9 & 24.0 & 49.9 & 0.9 & 24.5 \\
\hline $\mathrm{p}$ & & 1.89 & 53.3 & 400 & 17.8 & 0.6 & 16.8 & 19.7 & 0.6 & 17.9 \\
\hline${ }^{4} \mathrm{He}$ & $\max$ & 0.142 & 9.3 & 924 & 3.6 & 0.2 & 8.79 & 3.5 & 0.2 & 9.06 \\
\hline${ }^{3} \mathrm{He}$ & & 0.0236 & 8.0 & 4804 & 0.45 & 0.03 & 2.77 & 0.44 & 0.03 & 2.9 \\
\hline total & & 2.06 & 70.6 & - & 21.8 & 0.6 & 19.2 & 23.7 & 0.6 & 20.3 \\
\hline
\end{tabular}




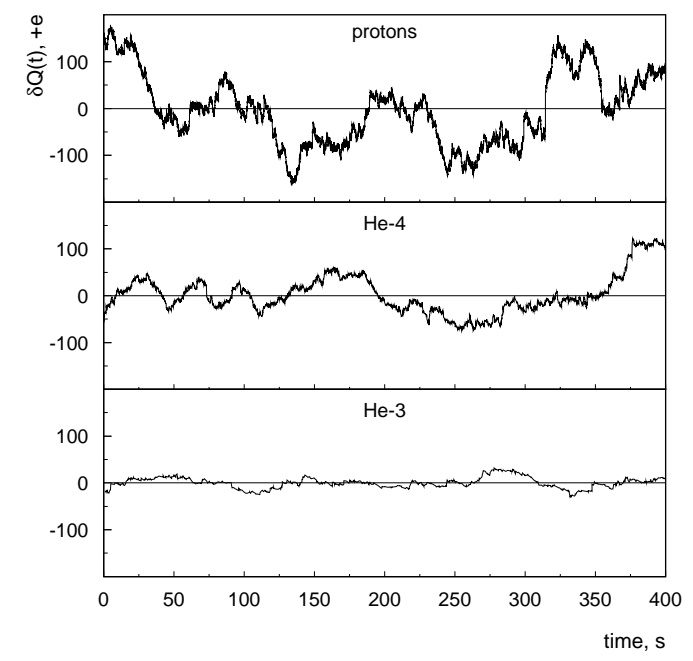

Fig. 4. Statistical fluctuations of the time-dependent net charge on $\mathrm{TM}_{0}$ around the average net charging rate for solar minimum conditions.

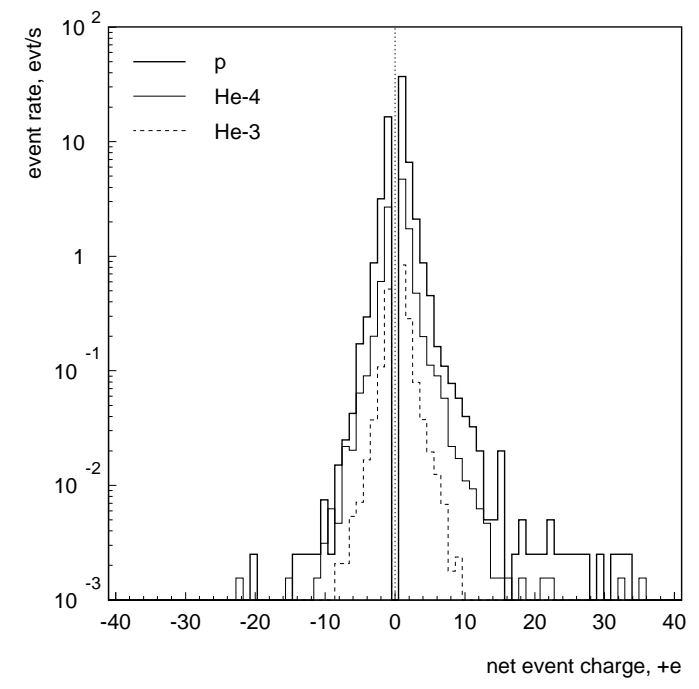

Fig. 5. Charge spectrogram for $\mathrm{TM}_{1}$ at solar minimum.

charging currents is represented in the spectrogram shown in Fig. 5. Although there is a degree of balance between negative and positive net charges, the latter dominate to produce a net positive rate. A few events were recorded with even larger net charges than Fig. 5 may suggest. The largest found in the 6 datasets was $+80+$ e, resulting from a $7 \mathrm{GeV}$ proton; in another event, over 200 electrons entered one TM leaving a net charge of $-20+\mathrm{e}$.

The shot noise for a particle rate $R_{q} / q e$ has a single-sided spectral density equal to $S_{q}=\left(2(q e) R_{q}\right)^{1 / 2}\left[+\mathrm{e} / \mathrm{s} / \mathrm{Hz}^{1 / 2}\right] . S_{R}$ is obtained by adding $S_{q}$ in quadrature over all values of $q$. The result is $24+\mathrm{e} / \mathrm{s} / \mathrm{Hz}^{1 / 2}$ and $20+\mathrm{e} / \mathrm{s} / \mathrm{Hz}^{1 / 2}$ for solar minimum and solar maximum, respectively. Finally, the charge fluctuations at frequency $f$ are obtained by integrating $S_{R}$ in the time domain: 
$S_{Q}(f)=S_{R} / 2 \pi f$

The charging noise can also be described by an 'effective rate' of single charges required to produce the same spectral density, $R_{e f f}=S_{R}^{2} / 2$. The solar maximum and minimum effective rates are $196+\mathrm{e} / \mathrm{s}$ and $288+\mathrm{e} / \mathrm{s}$, respectively. The latter figure is also twice that previously calculated in Ref. [6].

\subsection{Charging as a Function of GCR Energy}

Cosmic rays contribute to the overall charging rate according to their energy in the way shown in Fig. 6; also plotted are the GCR proton spectra (now in relative flux per histogram bin, not per unit energy). To a first approximation, the charging spectrum follows the respective input spectrum above $\sim 100 \mathrm{MeV} / \mathrm{n}$ for protons as well as He nuclei, and a factor $\simeq 2$ ratio of solar minimum to solar maximum rates reflects approximately the ratio between the GCR fluxes. Yet, the two scenarios have distinct spectral features: apart from the shift towards higher energies caused by the harder solar maximum flux, a peak is visible at lower energies in the solar minimum situation.
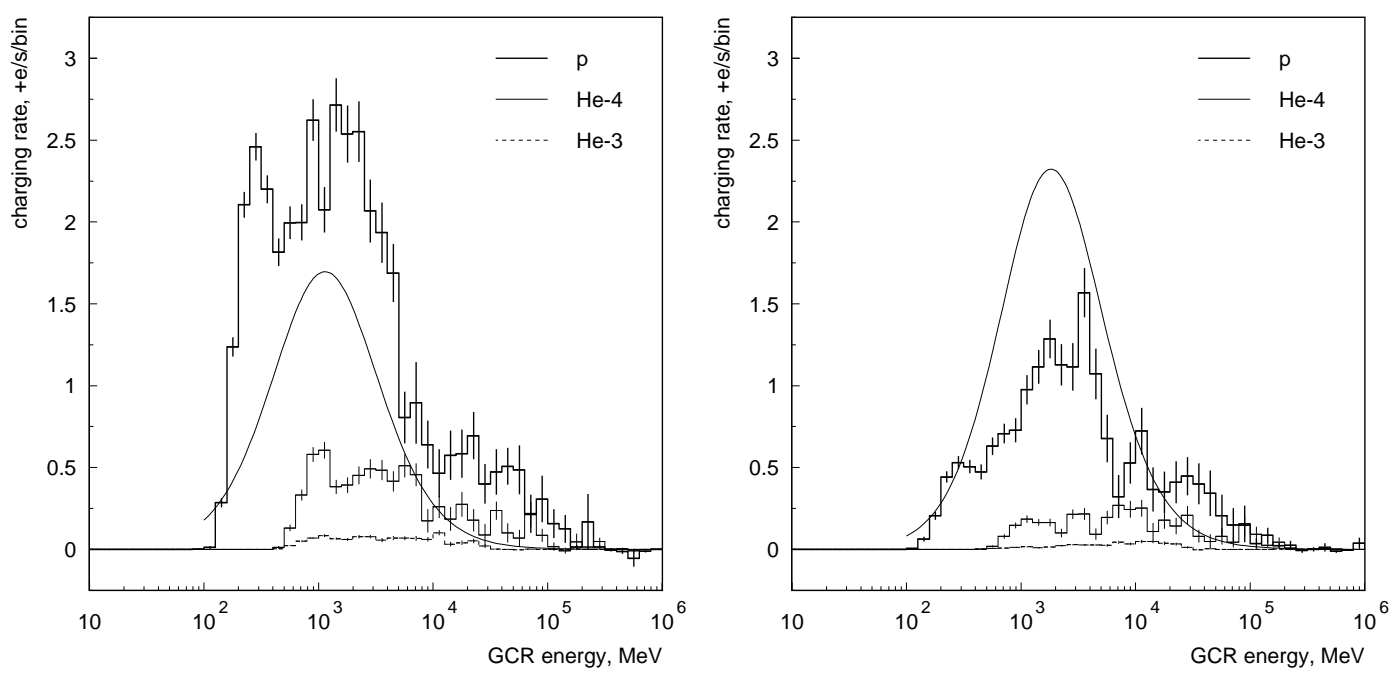

Fig. 6. Charging rate of $\mathrm{TM}_{0}$ as a function of primary energy at solar minimum (left) and solar maximum (right); the smooth lines represent the GCR proton spectra (frequency per histogram bin, on different scales).

Fig. 7 decomposes the effect of solar minimum protons according to the actual net charge stopping and being ejected from the TM. The feature at a few hundred $\mathrm{MeV}$ is readily explained in terms of single positive charges stopping in the TM, which are mostly protons. A mass overburden of at least $10-15 \mathrm{~g} / \mathrm{cm}^{2}$ encloses the test masses, which shields against primaries below $100 \mathrm{MeV} / \mathrm{n}$. The test cube itself represents $90 \mathrm{~g} / \mathrm{cm}^{2}$ — it takes a $\sim 400 \mathrm{MeV}$ proton to 
get through it. The rate of 100-400 MeV protons directly stopping in the TM is approximately $10+\mathrm{e} / \mathrm{s}$ at solar minimum (25\% of the proton-induced rate) and negligible for the harder solar maximum spectrum.

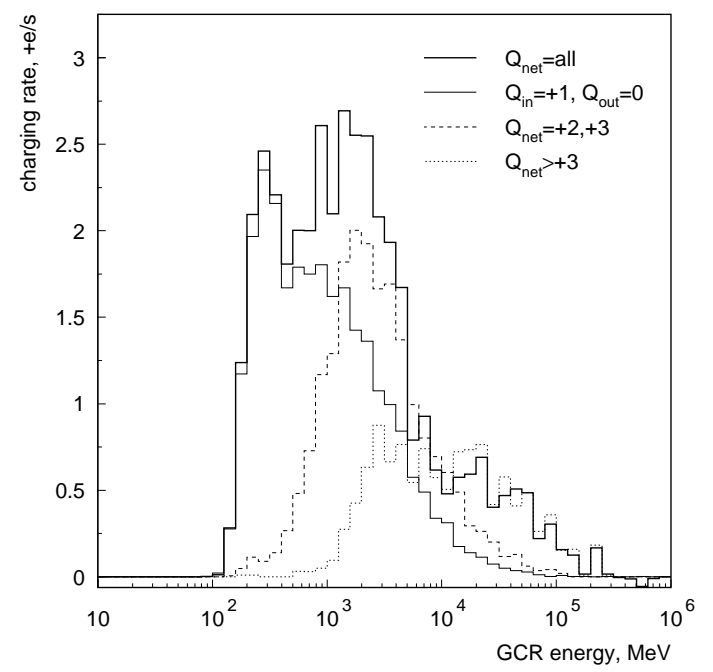

Fig. 7. Analysis of the proton charging rate for $\mathrm{TM}_{0}$.

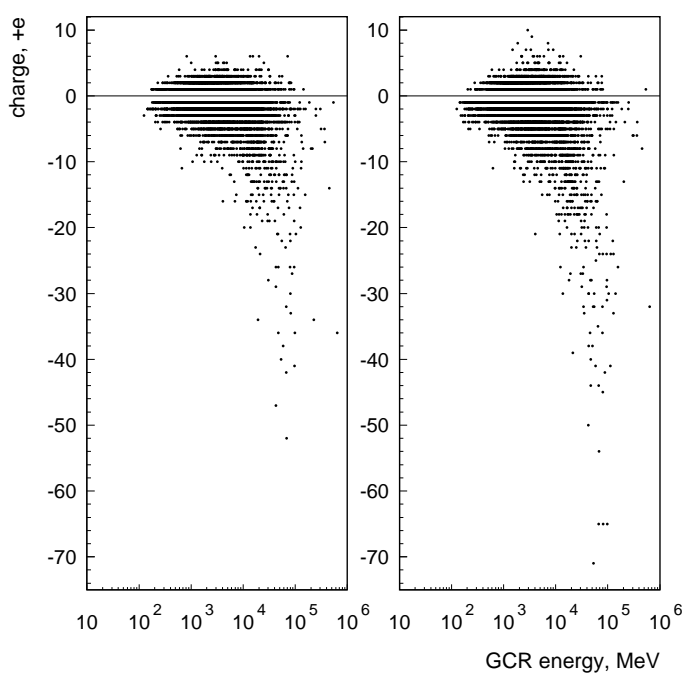

Fig. 8. Distribution of charges entering (left) and leaving (right) $\mathrm{TM}_{0}$ as a function of primary energy for solar minimum protons.

Increasingly energetic primaries result in higher net charges, as $\mathrm{e}^{+} / \mathrm{e}^{-}$pair production and cascaded nuclear reactions take over. This is illustrated in Fig. 8, which shows the charges stopping and being ejected from the TM as a function of primary energy - only for those events producing a net result. High-energy events are quite complicated, occasionally leading to 100 negative particles (mostly electrons) stopping in the TM while comparable numbers are ejected from it. In spite of some cancellation, the net charging is positive. Secondary electrons are responsible for a charging rate of $+28+\mathrm{e} / \mathrm{s}$ for solar minimum protons (almost $70 \%$ of the proton-induced rate). 
Although electromagnetic showers can create many $\mathrm{e}^{+} / \mathrm{e}^{-}$pairs and gamma rays, nuclear reactions are the most important charging mechanism at higher energies, effectively multiplying the number of primaries in the spacecraft. This multiplication role of hadronic interactions was confirmed by a simple simulation carried out with a basic geometry of concentric spheres providing $13 \mathrm{~g} / \mathrm{cm}^{2}$ of shielding to one cubic TM. The charging rate induced by solar minimum protons suffering electromagnetic processes alone was almost 3 times smaller than that obtained when hadronic processes were added. This fact partly explains the higher rates obtained in the present work.

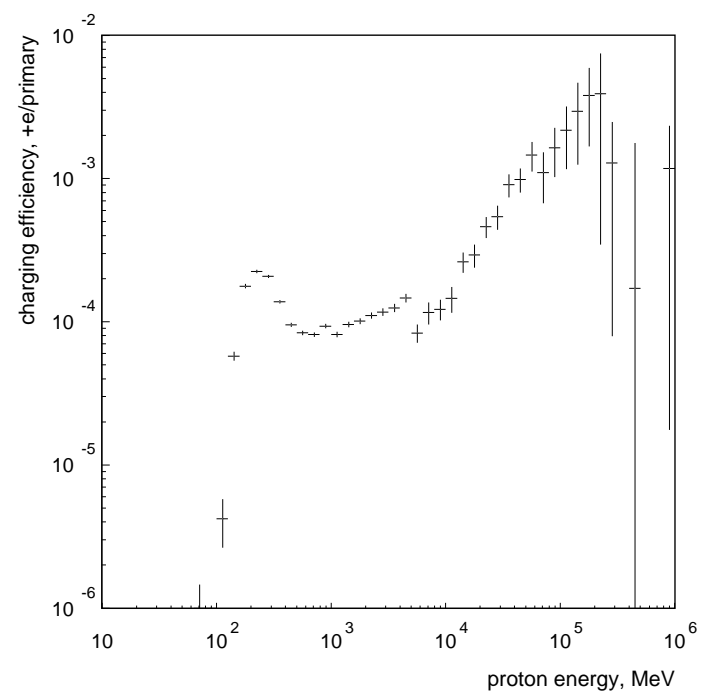

Fig. 9. Spectral charging efficiency for protons.

By dividing the charging spectrum by the input flux one obtains a spectral charging efficiency, $Q\left(E_{0}\right) / N_{0}\left(E_{0}\right)$, shown in Fig. 9 for protons. The charging threshold at $100 \mathrm{MeV}$ is clearly seen in the figure, as is the initial peak previously explained in terms of protons stopping in the TM. In this region, and up to approximately $10 \mathrm{GeV}$, the charging efficiency is $10^{-4}$ positive charges for each proton randomly emitted from the generator sphere. The discontinuity visible near $6 \mathrm{GeV}$ is artificially created by the change-over between inelastic scattering models for protons (intra-nuclear cascade and quark-gluon string models). At very high energies the charging efficiency decreases to zero (within errors). 


\section{Solar Energetic Particles}

\subsection{Characterisation of Solar Particle Events}

Irregular fluxes of solar particles (mainly protons) will add to the steady background of GCRs, leading to further charging of the LISA test masses. Solar events associated with radio bursts or moderate x-ray flares are relatively short-lived ( hours) and very frequent ( 1000/year). However, their energy spectrum is usually too soft $(<50 \mathrm{MeV})$ to penetrate the shielding overlying the TMs. A second kind of phenomena, known as solar energetic particle (SEP) events, are associated with shock acceleration in coronal mass ejections and their spectrum can extend up to hundreds of $\mathrm{MeV}$. Although much rarer than solar flares, SEP events can have large particle fluences above $100 \mathrm{MeV}$ and deposit significant amounts of charge over periods of days. It is important to characterise these events in terms of their frequency of occurrence, duration, peak flux and total fluence as well as energy spectrum in order to calculate their effects.

We have investigated the charging potential of two SEP events which can have operational implications for the LISA mission. Event 1 occurred on 29 Sept 1989 and is a well-known extreme event, with a fluence $\Phi_{40} \simeq 10^{9}$ protons $/ \mathrm{cm}^{2}$ above $40 \mathrm{MeV}$, which caused widespread instrument malfunction in space (and on Earth!). Event 2 is a small, unremarkable event registered on 20 May 2001, with a fluence of $\Phi_{40} \simeq 6 \times 10^{5}$ protons $/ \mathrm{cm}^{2}$. The respective proton fluxes recorded by the Geostationary Operational Environmental Satellites (GOES) [20] are shown in Fig. 10.

The Weibull function is found to describe many SEP energy spectra. The Weibull differential flux (in protons $/ \mathrm{cm}^{2} / \mathrm{s} / \mathrm{sr} / \mathrm{MeV}$ ), is:

$$
\phi(E)=A k \alpha E^{\alpha-1} \exp \left(-k E^{\alpha}\right),
$$

where $E$ is the primary energy and $A, k$ and $\alpha$ are free parameters. Event 1 has been fitted with such a function using data from space-borne detectors and ground-level neutron monitors together with radiation transport codes [21]. This fit is shown in Fig. 11. The peak fluxes in three GOES channels are also plotted in the figure.

Events with small fluence are usually not amenable to a similar parameterisation. Nevertheless, Event 2 was fitted with a Weibull function with the same spectral indices ( $\alpha$ and $k$ ) found for Event 1 but with $A$ scaled to agree with the GOES data. This approach should be sufficient for the estimation of charging rates. The result is also shown in Fig. 11. Note that this spectrum excludes 

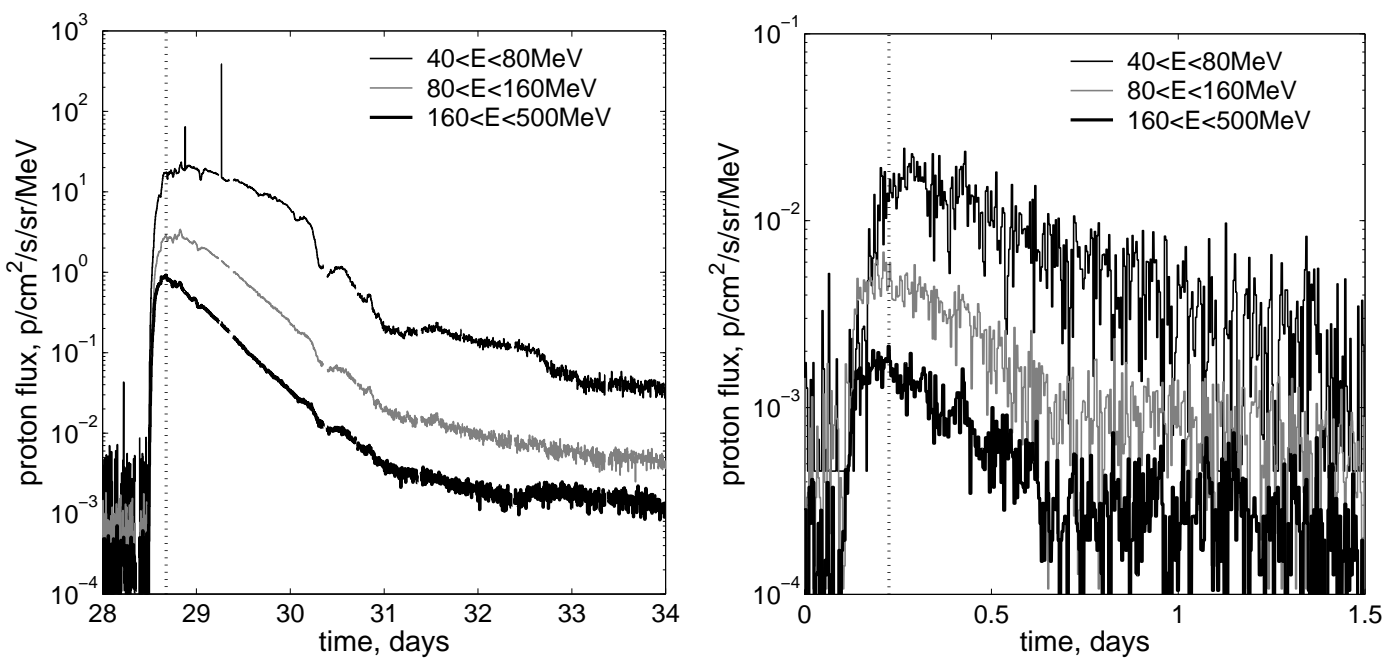

Fig. 10. Timelines of proton fluxes in three energy ranges measured by GOES satellites for Event 1 (29 Sept 1989) and Event 2 (20 May 2001). Note that the fluxes preceding the two events are higher than expected for solar maximum. The time at which the peak flux in the highest energy band is reached is also shown.

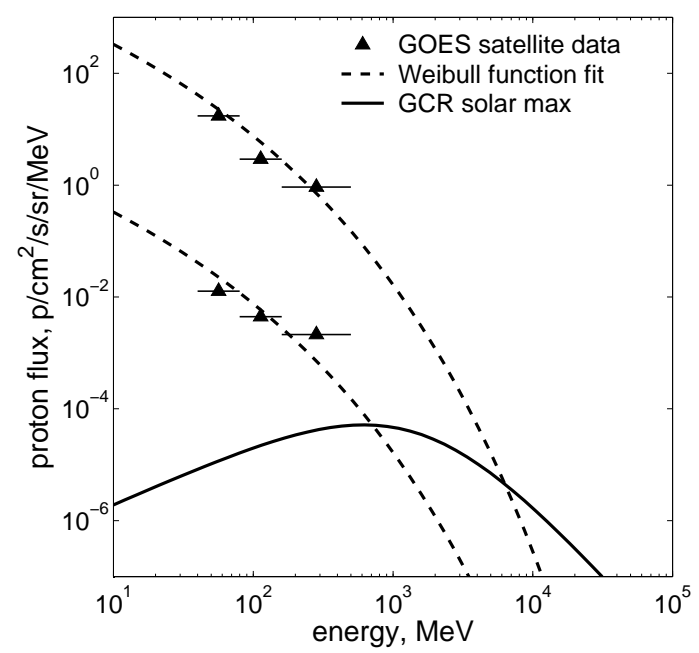

Fig. 11. Proposed differential energy spectra at peak flux for Event 1 and Event 2 using a Weibull function fit and the GOES data. Also shown is the spectrum for GCR protons at solar maximum.

the GCR background, which should be added in charging calculations.

Finally, the SEP event rate must be established from the relative distribution of event sizes together with the predicted rates for the occurrence of a particular event fluence. The well-known Nymmik model, based on a statistical analysis of historical records, applies to event fluences $\Phi_{30}(E>30 \mathrm{MeV})$ in the range $10^{5}-10^{11}$ protons $/ \mathrm{cm}^{2}$ [22]. In this model, the mean occurrence frequency, $\langle N(t)\rangle$ (events/year), of SEP events with fluence $\Phi_{30}>10^{5}$ protons $/ \mathrm{cm}^{2}$ is 
a function of solar activity through the average monthly sunspot number, $W$ :

$$
\langle N(t)\rangle=0.3 W(t)^{0.75}
$$

In the same study, the distribution of event fluences is fitted with a power law:

$$
\frac{1}{\langle N\rangle} \frac{d N\left(\Phi_{30}\right)}{d \Phi_{30}} \propto \Phi_{30}^{-1.41}
$$

These equations allow us to estimate the SEP event rate shown in Fig. 12. A sunspot number $W=100$ was considered to be representative of the solar maximum conditions prevailing around 2012 when LISA is scheduled for launch. This analysis confirms that events as large as Event 1 occur at most once in the 11-year solar cycle (note that $W$ decreases either side of solar maximum). Smaller ones such as Event 2 should occur some 6 times per year. The total number expected near solar maximum is of order 10. In Fig. 13 we present a projection of events expected during the mission lifetime. The same figure plots the probability for a SEP fluence $\Phi_{30}>10^{5}$ protons $/ \mathrm{cm}^{2}$ to occur in a $10^{6}$ s period, assuming a Poisson distribution with mean given by Eq. $2(W(t)$ is calculated from the average of 13 solar cycles). Such period represents a hypothetical extension of the LISA bandwidth presently under discussion. This calculation suggests that such a long dataset can easily be contaminated by a solar event.

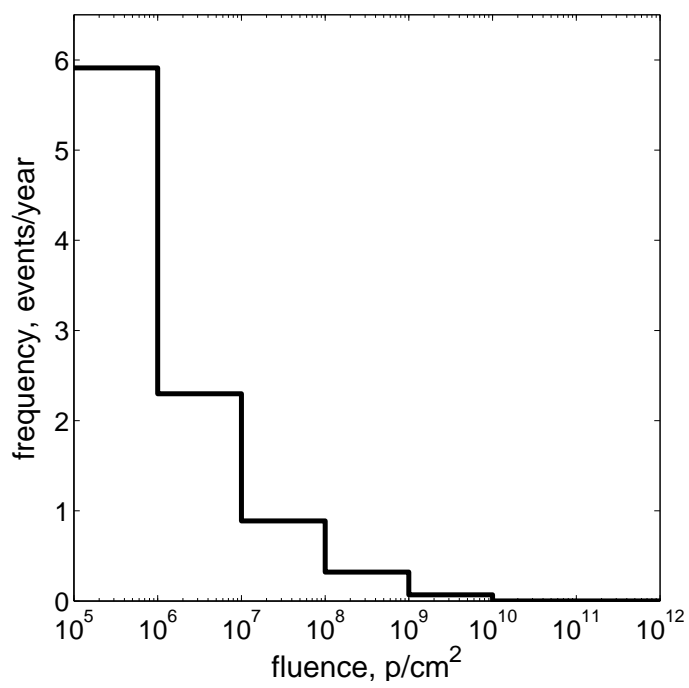

Fig. 12. SEP event frequency distribution as a function of event fluence for an average sunspot number $W=100$. 


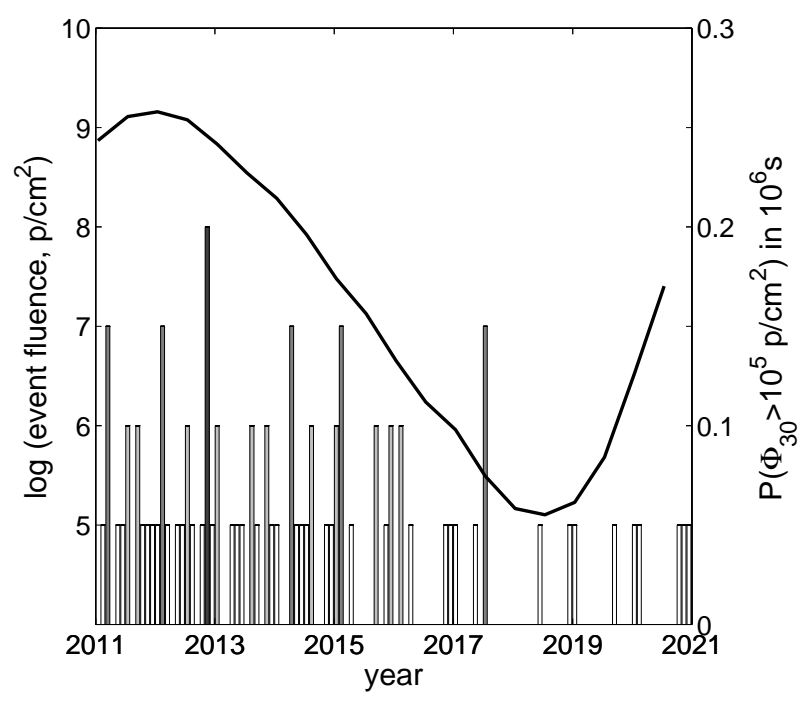

Fig. 13. Histogram: Predicted scenario of SEP events for the lifetime of the LISA mission. Line: Probability for a fluence $\Phi_{30}>10^{5}$ protons $/ \mathrm{cm}^{2}$ to occur during a $10^{6} \mathrm{~s}$ period along the solar cycle.

Table 2

Charging from SEP Events

\begin{tabular}{lcc}
\hline & $\begin{array}{c}\text { Event 1 } \\
29 \text { Sept 1989 }\end{array}$ & $\begin{array}{c}\text { Event 2 } \\
20 \text { May 2001 }\end{array}$ \\
\hline$\Phi_{30}$, protons $/ \mathrm{cm}^{2}$ & $1 \times 10^{9}$ & $6 \times 10^{5}$ \\
event duration, days & $\sim 5$ & $\sim 1$ \\
decay time, days & 0.43 & 0.34 \\
rate at peak flux, $+\mathrm{e} / \mathrm{s}$ & 68,000 & 87 \\
charging from SEP,$+\mathrm{e}$ & $3 \times 10^{9}$ & $3 \times 10^{6}$ \\
charging from GCR, $+\mathrm{e}$ & $1 \times 10^{7}$ & $2 \times 10^{6}$ \\
\hline
\end{tabular}

\subsection{Charging Rates from SEP Events}

The charging expected from these SEP events is obtained by multiplying the differential energy spectra by the spectral charging efficiency previously shown in Fig. 9. The charging spectrum for Event 2 added to solar maximum protons is shown in Fig. 14. The charging rate at peak flux is estimated at $87 \pm 3+\mathrm{e} / \mathrm{s}$ (the error reflects only the MC uncertainty). This is some 4 times above the solar maximum rate. On the other hand, Event 1 could charge the LISA test masses at $\sim 70000+\mathrm{e} / \mathrm{s}$ at peak flux! Considering a simple exponential decay of the fluxes shown in Fig. 10, we obtain the total charge deposits indicated in Table 2. 


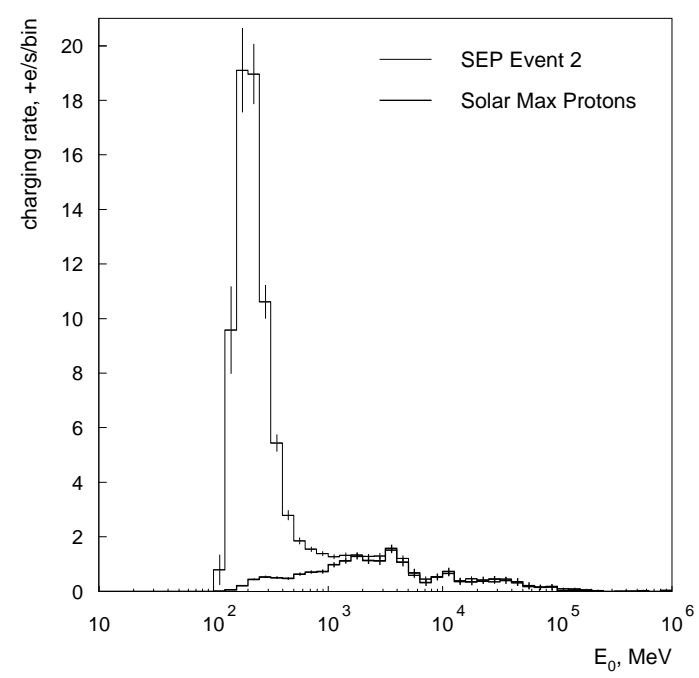

Fig. 14. Charging spectra for small SEP event and GCR protons at solar maximum.

This analysis is reassuring in that the number of SEP events with the potential to produce spurious signals is relatively small even at the peak of solar activity. This situation can change in periods of exceptional solar turbulence $(W=200$ was observed in the most intense solar cycle on record). The occurrence of solar events should be correlated with the science data. Proton monitors aboard the LISA spacecraft could be a very useful diagnostic tool for this purpose. Earthbased monitors (or those on other missions) may miss spatial effects only visible on the LISA constellation, or even on one of its spacecraft alone.

\section{Non-simulated Physics}

The MC simulation was complemented by an assessment of other physics processes for their potential for TM charging. We categorise these into kinetic emission from surfaces, photon emitting processes and isotope production in the IS. The starting point for this analysis is the MC calculation of the primary and secondary particle fluxes tallied at the surface of the TMs. The latter are henceforth considered to be made entirely from gold.

\subsection{Kinetic Emission Processes}

Low energy electrons $(\sim \mathrm{eV})$ are emitted from surfaces bombarded by electrons and ions. For fast (but non-relativistic) projectiles this phenomenon is often known as kinetic emission, usually abbreviated as EIEE or IIEE for electron- and ion-induced electron emission, respectively. EIEE is exploited, for example, in scanning electron microscopy (SEM) and electron multipliers. 
Useful reviews are available on this subject $[23,24,25]$ — although somewhat outdated in terms of experimental data. The analysis presented here includes data more relevant to the case of gold surfaces and high primary energies. Normal incidence is assumed for simplicity.

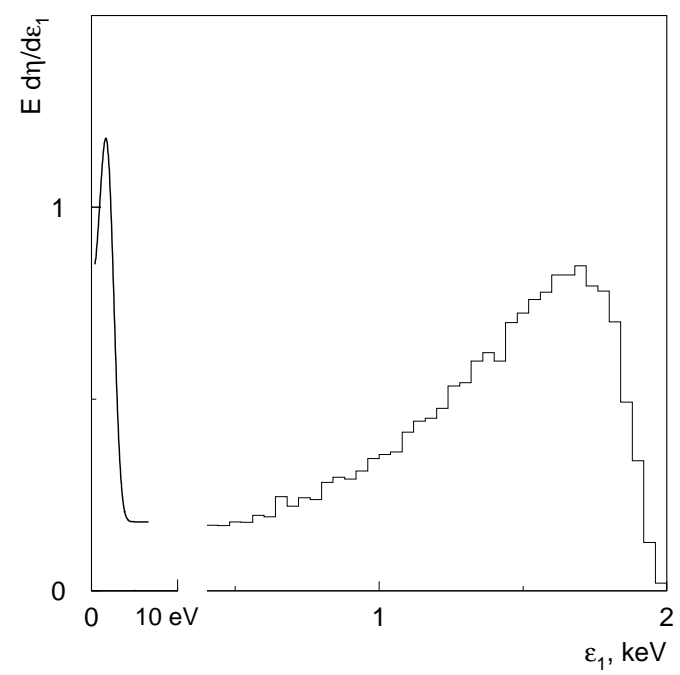

Fig. 15. Illustration of the electron spectrum emitted from a gold surface irradiated by $2 \mathrm{keV}$ electrons at normal incidence. Thin line: backscattered primaries (Geant4 simulation); Thick line: kinetic electron emission.

In EIEE, the energy spectrum of electrons emitted from the solid extends from close to the incident electron energy, $E$, down to less than $1 \mathrm{eV}$, and has the generic shape illustrated in Fig. 15. The higher-energy part of the spectrum is due to backscattered primaries, the total number of which is characterised by a reflection yield, $\eta$. This process is reproduced by Geant4. A significant electron population is also represented in the figure at energies below $\sim 10 \mathrm{eV}$. (Note that the total yield can be greater than 1) These are secondary electrons created by the incident particle which find their way to the solid surface. An electron is emitted if its kinetic energy perpendicular to the boundary is greater than the surface barrier (for metals, this corresponds to the work function, $\phi)$. This secondary electron yield, $\delta$, has a very soft spectrum, peaking at a few $\mathrm{eV}$. This peak is really due to the opposing trends of the delta-ray production spectrum and the work function subtraction at the surface, and has no particular meaning beyond that. Naturally, the secondary electron yield is not taken into account in the simulation, and has to be assessed separately.

Fast ions, such as $\mathrm{H}$ and He nuclei, also lead to electron emission (IIEE). In this instance the backscattering probability is small, but the kinetic emission process is otherwise very similar to EIEE. An estimate of this contribution to TM charging is also required.

In addition, one must consider secondary emission from transmitted electrons and ions, i.e. those ejected forward from the electrodes and from the TMs 
by primaries travelling from the solid into vacuum. Accurate experimental information on these forward yields is scarce, but there is indication that they are similar to the backward yields [26].

Electrons emitted by EIEE and IIEE are generated extremely close to the surface of the solid, in a layer $\lesssim 50 \AA$ thick. This effect is therefore very sensitive to the surface conditions. Oxide layers on metals and semiconductors are expected to increase the yield since insulators usually have a higher emission yield than metals, although different metals were observed to change in different directions when exposed to oxygen [27]. No change was observed for $\mathrm{Au}$ targets in the same study. Nevertheless, the possible adsorption of foreign species at the surface, namely organic deposits, should be considered.

\subsubsection{EIEE and IIEE yields}

According to the theory of Schou [28], which explores the similarity between kinetic emission of electrons and atom sputtering, the secondary yield caused by an incident non-relativistic electron with energy $E$ is given by:

$$
\delta_{P E}=\beta^{\star} S_{e}(E) \Lambda
$$

where $S_{e}(E)$ is the electronic stopping power at the primary electron energy $E$, the parameter $\Lambda$ depends only on the target material and $\beta^{\star}$ is a dimensionless function almost insensitive to energy. This proportionality of the secondary electron yield to the electronic stopping power has long been recognised. This treatment ignores the contribution of secondaries generated by backscattered electrons, which must be accounted for [29]:

$$
\delta=\delta_{P E}(1+\beta \eta)
$$

In this expression, $\beta$ is the ratio of the mean secondary electron generation of one backscattered electron to that of one primary electron and $\eta$ is the backscattering coefficient.

The parameters $\delta_{P E}$ and $\beta$ in Eq. 5 have been measured for thin gold films for electron energies in the range $10-100 \mathrm{keV}$ [26]. In addition, we consider $\eta=0.5$ in this energy range, in order to add the contribution of secondaries produced by backscattered primaries to the experimental values. Experimental data were also found for lower energies [30], which reveal a total electron yield peaking at $\simeq 2$ near $1 \mathrm{keV}$. These data include reflected primaries, which are a sizeable fraction even at $1 \mathrm{keV}$. Backscattering has already been modelled by Geant4, and must therefore be subtracted. The two datasets are shown in Fig. 16 a), along with the stopping power for electrons in gold over a wide energy range [31]. Curves 2 and 3 show our corrected estimates for $\delta$. There is 
fair agreement between the energy dependence of the two datasets and that of the stopping power above $\sim 1 \mathrm{keV}$ (the abrupt behaviour of $S_{e}$ at $\sim 100 \mathrm{eV}$ is due to the particular model considered [32]). We have adopted a secondary electron yield, $\delta$, which attempts to reproduce the experimental data whilst following the energy dependence of the stopping power in the non-relativistic Bethe-Block region (10 keV-1 MeV). This is indicated by curve 4 in Fig. 16 a).
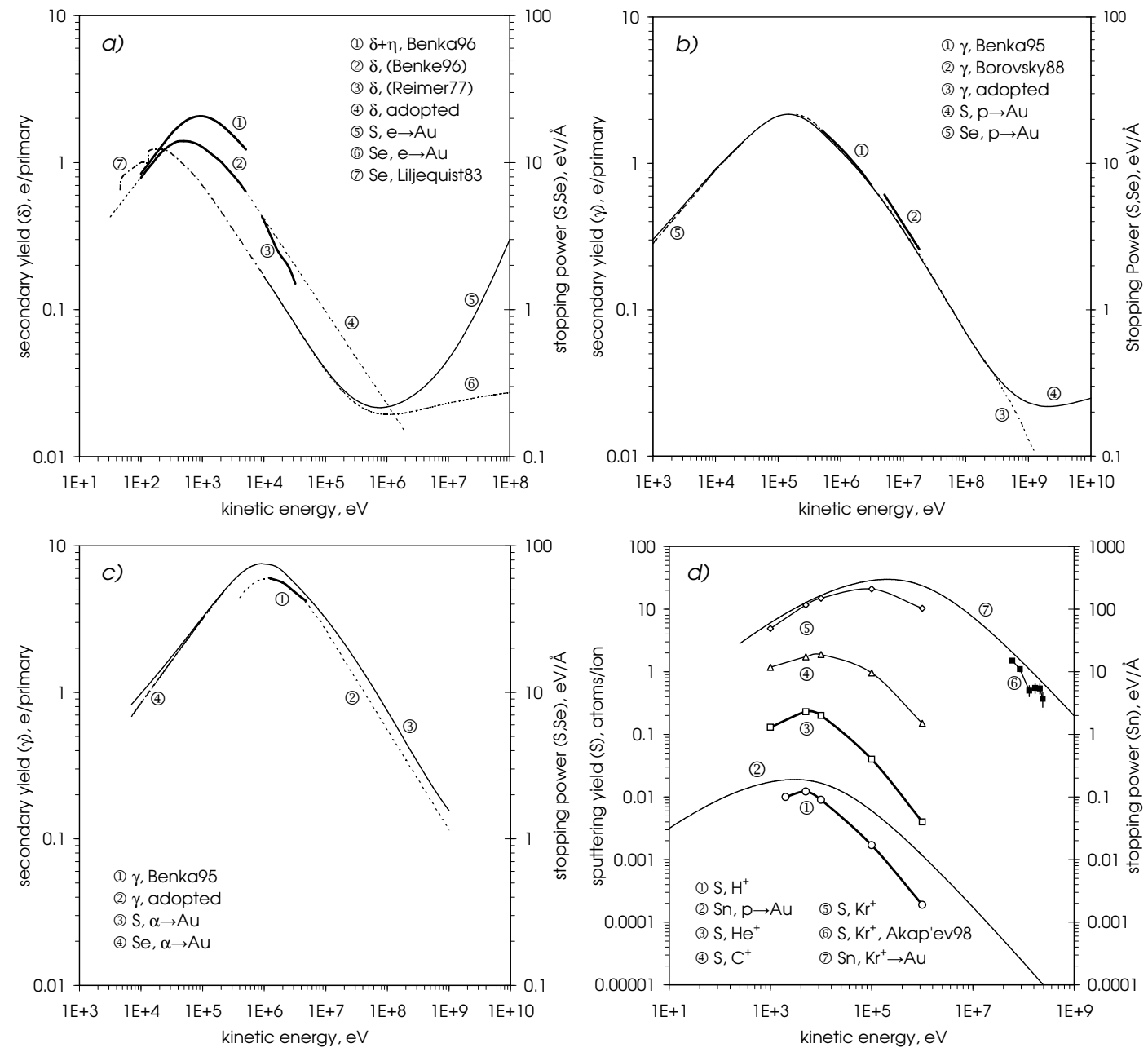

Fig. 16. a) EIEE yield and stopping power for electrons in gold. b) IIEE yield for protons. c) IIEE yield for alpha particles. d) Sputtering yield and nuclear stopping powers for several projectiles in gold.

The theory of ion-induced emission is simpler than that of EIEE since primary backscattering is not significant. The ion-induced emission yield, $\gamma$, of secondary electrons by fast, non relativistic ions is approximately proportional to the electronic component of the stopping power for the ion in the material. Proton yields are comparable to those of electrons of similar speed. To obtain a charging rate estimate we make a similar extrapolation of low energy experimental measurements to the relevant part of the primary projectile spectrum. 
Note that this dependence is being extrapolated to relativistic energies, which are beyond the applicability of the theory.

Fig. 16 b) shows the adopted yields for protons based on data found in Ref. [33] and Ref. [34]. Here too the energy dependence of the yield is in good agreement with that of the stopping power over the energy range of the measurements. Multiply-charged ions have larger yields, reflecting higher rates of energy loss in the material. At GCR energies the helium flux is composed of $\mathrm{He}^{2+}$ rather than singly-ionised atoms. Fig. 16 c) shows the adopted yield based on the data from Ref. [33]. Note that $\delta \simeq 6$ at the peak of the stopping power.

\subsubsection{Kinetic emission rates}

The energy spectra of electrons and protons tallied at the TM surface regardless of whether or not they lead to a net event charge - are shown in Fig. 17. Similar data were generated for solar maximum protons as well as ${ }^{4} \mathrm{He}$ primaries. It is worth pointing out the noticeable discontinuity observed in the electron spectrum near $800 \mathrm{eV}$, which is due to the limitation of the protoninduced delta-ray production model in Geant4 mentioned in Section 2.3. A large uncertainty should therefore be attributed to the sub-keV part of the energy spectra, as it is difficult to estimate the number of electrons suppressed by the cut (although no major error in the charging rate is expected).
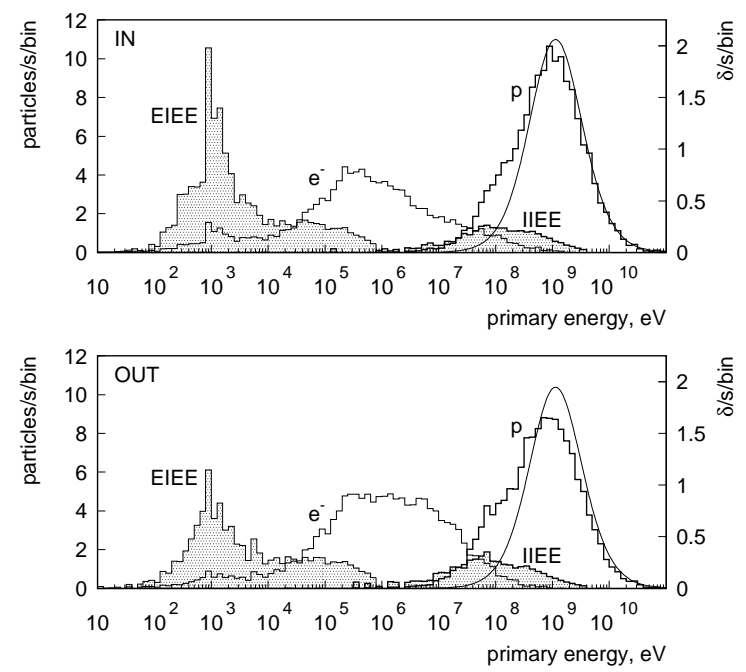

Fig. 17. Spectrum of electrons and protons entering (top) and leaving (bottom) one TM for incident protons at solar minimum and respective kinetic emission rates (shaded).

On the same plot we show the corresponding emission rates obtained by multiplying the primary spectra by the normal incidence yields. The net rates for primaries entering and leaving the TMs are summarised in Table 3. If identi- 
Table 3. Estimates of EIEE and IIEE rates.

\begin{tabular}{|c|c|c|c|c|c|c|c|c|c|c|}
\hline $\begin{array}{l}\text { primary } \\
\text { particle }\end{array}$ & $\begin{array}{c}\text { solar } \\
\text { activity }\end{array}$ & $\begin{array}{l}\text { elect } \\
\mathrm{e} / \mathrm{s}\end{array}$ & $\delta / \mathrm{s}$ & $\begin{array}{l}\text { elect } \\
\mathrm{e} / \mathrm{s}\end{array}$ & $\begin{array}{l}\delta / \mathrm{s} \\
\delta \text { out }\end{array}$ & $\begin{array}{l}\text { hadr } \\
\text { h/s }\end{array}$ & $\begin{array}{l}\text { ns in } \\
\gamma / \mathrm{s}\end{array}$ & $\begin{array}{l}\text { hadr } \\
\text { h/s }\end{array}$ & $\begin{array}{l}\text { ns out } \\
\gamma / \mathrm{s}\end{array}$ & $\begin{array}{c}\langle\text { K.E. }\rangle \dagger \\
+\mathrm{e} / \mathrm{s}\end{array}$ \\
\hline $\mathrm{p}$ & & 112 & 17.6 & 141 & 14.0 & 154 & 4.7 & 145 & 5.5 & 20.9 \\
\hline${ }^{4} \mathrm{He}$ & $\min$ & 24 & 2.3 & 31 & 2.2 & 19 & 5.7 & 21 & 8.0 & 9.1 \\
\hline${ }^{3} \mathrm{He}$ & & - & - & - & - & - & - & - & - & - \\
\hline total & & 136 & 19.9 & 172 & 16.2 & 173 & 10.4 & 166 & 13.5 & 30.0 \\
\hline $\mathrm{p}$ & & 72 & 12.0 & 94 & 8.7 & 80 & 2.1 & 84 & 3.0 & 12.9 \\
\hline${ }^{4} \mathrm{He}$ & $\max$ & 14 & 1.2 & 19 & 1.3 & 10 & 3.4 & 17 & 4.7 & 5.3 \\
\hline${ }^{3} \mathrm{He}$ & & - & - & - & - & - & - & - & - & - \\
\hline total & & 86 & 13.2 & 113 & 10.0 & 90 & 5.5 & 101 & 7.7 & 18.2 \\
\hline
\end{tabular}

$\bar{\dagger}$ Average kinetic emission from the TM and the inner electrode housing. 
cal yields are considered for both incident and transmitted particles (forward and backward emission), and also for the TM as well as the electrode surfaces, then these four contributions should nearly cancel out statistically. However, this cancellation can be compromised by sensing and actuating voltages applied to the electrodes. Even if the net rates do cancel out, the stochastic nature of the emission will be a source of spurious acceleration noise in the sensor. For this reason, Table 3 also indicates the average emission rates $\left({ }^{1} / 2(\delta+\gamma)_{\text {IN }}+{ }^{1} / 2(\delta+\gamma)_{\text {OUT }}\right)$, which we take to represent the importance of this effect (and should not be added to $R$ as a net charging rate). The average rate for solar minimum is $30+\mathrm{e} / \mathrm{s}$, a figure comparable with the $\mathrm{MC}$ rate and larger than He-induced charging.

\subsubsection{Atom sputtering}

Sputtering is the ejection of atoms from a surface under bombardment from energetic ions. Although similar in nature to kinetic electron emission, this is mainly a momentum-transfer process between nuclei. Consequently, it depends on the nuclear stopping power, $S n$, rather than on the electronic component. Although sputtered species are likely to be electrically neutral (although this is not always the case) charge emission may occur from surfaces struck by the sputtered atoms.

The sputtering yields of gold surfaces - the number of atoms ejected per incident ion — were obtained with the SRIM2003 package [35], a software widely used for calculation of ion interactions in matter. SRIM reproduces the experimental yields found in Ref. [36] for bombardment of $\mathrm{Au}$ with singlyionised noble gases at 1-100 keV energies. The sputtering yields, $S$, for $\mathrm{H}, \mathrm{He}$, $\mathrm{C}, \mathrm{Fe}$ and $\mathrm{Kr}$ ions were then calculated, using a full-cascade simulation, at ion kinetic energies between $1 \mathrm{keV}$ and $1 \mathrm{MeV}$. These are shown in Fig. $16 \mathrm{~d}$ ). Carbon is the third most abundant GCR species, after $\mathrm{H}$ and He.

The nuclear stopping powers for protons and krypton ions in gold are also shown in the figure. Experimental data were found for krypton above $\sim 100$ $\mathrm{MeV}$ [37]. For this projectile, an extrapolation of $S$ to high energies based on $S n$ seems justified. Adopting a similar trend for the lighter projectiles allows us to conclude that sputtering rates are negligible, much smaller than 1 atom/s. They can therefore be safely ignored.

\subsection{Photon Emission Processes}

We now examine non-simulated photon processes which can contribute to TM charging. In this category we include x-ray transition radiation (XTR), $\mathrm{x}-$ ray Cherenkov radiation (XCR) and particle-induced x-ray emission (PIXE). 
Their effects may be two-fold: electron emission can be due to self-absorption within the bulk material or photoemission from a surface across the sensor gaps.

A surface irradiated by x-rays emits a primary spectrum of fast photoelectrons and Auger electrons as well as low energy $(\mathrm{eV})$ secondaries generated by kinetic emission. The quantum efficiency (QE) of gold surfaces can be as high as $10 \%$ for photon energies in the range $20 \mathrm{eV}-10 \mathrm{keV}$ [38]. Secondary electron emission dominates the total QE in most materials. For gold, the primary spectrum constitutes only 3-20\% of the total yield over that energy range [39]. A rapid decrease in efficiency is observed into the UV region, as the photon energy approaches the surface work function. An electron yield of $\sim 10^{-5}$ electrons per absorbed photon is expected at $5 \mathrm{eV}[40]$. The low UV QE abates our concern with sources of optical emission, such as optical transition radiation (OTR) and optical Cherenkov emission (OCR).

\subsubsection{Proton-induced $x$-ray emission}

The ionisation cross-section of a particular atomic shell increases with projectile energy in the $\mathrm{MeV}$ range, peaking when the projectile velocity equals the electron velocity in that particular shell. A simple rule-of-thumb for the peak energy is $E_{0}=1840 A U_{i}$, where $A$ is the projectile mass number and $U_{i}$ is the electron binding energy [41]. In gold, this corresponds to proton energies of approximately $150 \mathrm{MeV}$ for the $\mathrm{K}$ shell $\left(U_{i}=80 \mathrm{keV}\right)$ and $25 \mathrm{MeV}$ for the $\mathrm{L}$ shell $(14 \mathrm{keV})$. The $\mathrm{K}$ shell fluorescence yield, $\omega_{K}$, is practically unity for $\mathrm{Au}$ (whereas $\omega_{L} \approx 0.35$ ), which means that nearly all K-shell ionisation ends up in x-ray emission rather than Auger electrons. Given the large primary energies involved, one must confirm that photon fluxes across the sensor gaps are small. Although a prototype PIXE model does exists in Geant4, it still reproduces experimental data poorly in the present version of the toolkit. Instead, we have estimated the emission yields of $\mathrm{K}$ and $\mathrm{L}$ x-rays for protons incident on a thick gold sample using the procedure described in Ref. [41].

For a proton of energy $E_{0}$ entering a sample at angle $\theta_{i}$, the PIXE yield, $Y$, radiated into a solid angle $d \Omega$ around an x-ray exit angle $\theta_{o}$ is given by:

$$
Y\left(E_{0}, \theta_{i}, \theta_{o}\right)=\frac{N_{A} \omega_{i} f_{\alpha}}{A} \frac{d \Omega}{4 \pi} \int_{E_{0}}^{0} \frac{\sigma(E) T(E)}{S(E)} d E .
$$

In this expression, $N_{A}$ is Avogadro's number, $\omega_{i}$ is the fluorescence yield for the $i$-th atomic shell, $f_{\alpha}$ is the fraction of fluorescence for a particular x-ray ( $\mathrm{K}_{\alpha 1}, K_{\alpha 2}$, etc.), $A$ is the molar mass of the material, $\sigma(E)$ is the ionisation cross-section for the shell in question, $S(E)$ is the proton stopping power and 
$T(E)$ describes the x-ray self-absorption in the sample:

$$
T(E)=\exp \left(-\int_{E_{0}}^{E} \mu \frac{\cos \left(\theta_{i}\right)}{\cos \left(\theta_{o}\right)} \frac{1}{S(E)}\right),
$$

where $\mu$ is the mass attenuation coefficient at the x-ray energy. The integrals in Eq. 6 and Eq. 7 must be computed numerically. The proton stopping power is approximated by a power law in the 1-1000 MeV range. For the $\mathrm{K}$ - and L-shell ionisation cross-sections, the parameterisation proposed in Ref. [42] is used. Although intended for lower energies, the cross-sections predicted for $\mathrm{Au}$ agree satisfactorily with experimental data for K-shell ionisation by $160 \mathrm{MeV}$ protons [43] and L x-ray production at $30 \mathrm{MeV}$ [44].

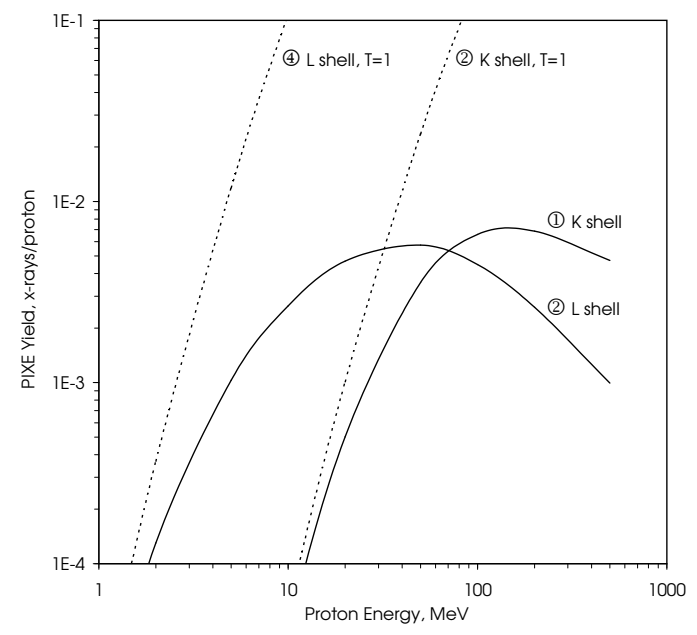

Fig. 18. K- and L-shell PIXE yields from protons on a thick Au target (normal incidence). The dotted lines describe the yields uncorrected for self-absorption $(T(E)=1)$.

The PIXE yields into the vacuum hemisphere are shown in Fig. 18 for normal incidence protons. This estimate indicates a value just under 0.01 photons/proton over the energy range of interest. Considering maximum QEs of 0.1 for L- and $\mathrm{K}$ x-rays, the proton rates given in Table 3 result in a charging rate below $1+\mathrm{e} / \mathrm{s}$. The photoelectron emission rate from $\mathrm{x}$-ray self-absorption in the sample itself is even smaller.

\subsubsection{Transition radiation}

Transition radiation (TR) consists of the emission of photons by fast charged particles crossing a boundary between two media with different dielectric constants. Optical photons are generated by slow, non- or slightly-relativistic incident particles (OTR), whereas ultra-relativistic projectiles produce preferentially x-rays (XTR). Although common radiators are dielectrics, any material 
boundary can generate TR photons, including metal/vacuum interfaces. In the latter case, large photon yields are not foreseen due to the opaque nature of the radiator. OTR is not expected to be an important source of TM charging due to both the very small photon absorption length and the relatively low photoelectric coefficients of gold at optical/UV wavelengths. X-ray emission should be estimated in view of the large flux of relativistic particles across the TM boundaries.

A particle with charge ze crossing from vacuum into a material with plasma frequency $\omega_{p}$ will produce a TR spectrum which diverges at low energies and decreases rapidly for photon energies above $\gamma \hbar \omega_{p}$, where $\gamma$ is the Lorentz factor. The number of photons generated above an energy $\hbar \omega_{0}$ is given by the following equation [45]:

$$
N \simeq \frac{\alpha z^{2}}{\pi}\left[\left(\ln \frac{\gamma \hbar \omega_{p}}{\hbar \omega_{0}}-1\right)^{2}+\frac{\pi^{2}}{12}\right]
$$

where $\alpha$ is the fine structure constant. As this equation suggests, the number of photons emitted above a fixed fraction of $\gamma \hbar \omega_{p}$ is constant. For a gold radiator $\left(\hbar \omega_{p} \simeq 8.5 \mathrm{eV}\right)$ the quantum yield above $0.1 \gamma \hbar \omega_{p}(\simeq 10 \mathrm{eV})$ for $10 \mathrm{GeV}$ incident protons is equal to $0.59 \% ; 10 \mathrm{MeV}$ electrons generate the same yield above $18 \mathrm{eV}$ TR energy. These small values are further attenuated by the opacity of gold at such low photon energies and the small QE of the gold surfaces opposite. No significant charging is thus expected from TR photons crossing the sensor gaps.

On the other hand, one can consider that most TR photons are absorbed in the solid and create photoelectrons very close to the surface, a fraction of which can be ejected - possibly accompanied by kinetic emission. Considering the TR and EIEE yields involved, together with the simulated particle fluxes across the TM boundary, we place an upper limit of $1+\mathrm{e} / \mathrm{s}$ for the charging contribution from transition radiation.

\subsubsection{X-ray Cherenkov radiation}

Cherenkov radiation (CR) is closely related to TR but, whereas the latter occurs at discontinuities of the dielectric constant, Cherenkov photons are emitted when the particle velocity is greater than the speed of light in the bulk medium. Optical Cherenkov emission (OCR) in transparent media is not important here for the reasons stated above for OTR. At higher energies, comparable to atomic levels, CR is absorbed over very short distances and so it manifests itself as ionisation created along the particle track. This is already included in the MC as a continuous energy loss term [46]. 
X-ray Cherenkov radiation (XCR) is suppressed in the limit of a transparent medium since photons emitted from different parts of the particle track interfere destructively, but this interference is broken by a finite photon attenuation length, $l$. The ceramic slabs supporting the electrodes, shown in Fig. 2, can act as XCR radiators. As in XTR, self-absorption near the surface is probably more important than the x-ray flux across the IS gaps.

We have estimated the number of Cherenkov x-rays emitted from the ceramic electrode supports into the forward hemisphere for $1 \mathrm{GeV}$ protons using the following yield equation [47]:

$$
\frac{\mathrm{d}^{3} N}{\hbar \mathrm{d} \omega \mathrm{d} x \mathrm{~d} \theta^{2}}=\frac{\alpha}{\pi \hbar c} \frac{\omega}{c} \theta^{2} \Im(Z),
$$

where $\hbar \omega$ is the photon energy, $x$ is the trajectory length, $\theta$ is the photon emission angle with respect to the particle trajectory and $Z$ is known as the complex formation zone of the medium: $Z=L /(1-i L / l)$, with:

$$
L=\frac{c}{\omega}\left[\gamma^{-2}+\frac{\omega_{p}^{2}}{\omega^{2}}+\theta^{2}\right]^{-1} .
$$

Note that a transparent medium $(l=\infty)$ implies $Z=L$, which eliminates the yield in Eq. 9. For the ceramics, the CR yield from a layer with thickness equal to $l \sim 0.1 \mathrm{~mm}$ is 0.025 photons/proton. Although only a fraction of the photoelectrons created over a thickness $l$ escapes the electrode surface, this process seems capable of TM charging at the level of $1+\mathrm{e} / \mathrm{s}$.

\subsection{Cosmogenic Activation}

The mechanisms analysed so far involve TM hits which happen, for all practical purposes, in coincidence with the primary cosmic ray. We now assess whether the activation of materials by GCR protons can lead to significant TM charging through the subsequent radioactive decay of relatively shortlived radioisotopes. The main concern is activation of the TM itself, since the IS housing provides enough shielding from external radioactive products.

The activity of a particular daughter species after an irradiation time $t$ is [48]:

$$
\mathcal{A}(t)=0.6 \frac{m}{A} \sigma \Phi\left(1-e^{-\lambda t}\right) \quad[\mathrm{Bq}],
$$

where $m / A$ is the dimensionless ratio of the total mass to atomic mass of the

material, $\sigma$ is the cross-section in barn, $\Phi$ is the proton flux in $\mathrm{p} / \mathrm{s} / \mathrm{cm}^{2}$ and 
$\lambda$ is the decay constant of the radioisotope. If the irradiation period is long compared to the half-life of a particular radioisotope, then the single-species activity in a 46-mm TM is given by $\mathcal{A}=6 \sigma \Phi$.

Radioisotope production by proton irradiation has been studied in the 100$2000 \mathrm{MeV}$ energy range for many targets [49]. For gold, the production crosssections are highest for daughter isotopes just below ${ }^{179} \mathrm{Au}$ (spallation) and decrease quickly for lighter elements. Overall, a dozen or so radioisotopes are produced at $\sim 0.1$ barn per nuclide; these decay quickly (relative to the mission duration) mainly by electron capture (EC). We estimate a secular-equilibrium activity of the order of $2 \mathrm{~Bq}$ for each spallation product. Only a very small fraction of these decays $(\sim 0.1 \%)$, which occur randomly in the TM volume, can actually eject charge from its surface. Therefore, this contribution to the charging rate can be safely ignored. Radioisotope production by neutron capture was also found to be similarly ineffective in TM charging.

\section{Conclusions}

The MC simulation of the interaction of GCR protons and He nuclei with the LISA spacecraft indicates TM charging rates of nearly $25 \mathrm{He} / \mathrm{s}$ for solar maximum conditions, rising to $50 \mathrm{He} / \mathrm{s}$ for solar minimum. Table 4 summarises the main results. We consider an additional error of $\pm 30 \%$ on the charging rates to account for uncertainties in the GCR spectra, physics models and geometry implementation. Although most of the charging is inflicted by protons, $\mathrm{He}$ contributes disproportionately to its abundance due to the double ion charge.

The solar minimum rate is almost twice that previously obtained with Geant4 [6], which is explained by the far more detailed geometry implementation and the use of more complex physics models. The new result is almost 5 times larger than the original Geant3 simulation [4]. Apart from the smaller $40 \mathrm{~mm}$ TM considered in that study, the difference is also explained by insufficient hadronic models in Geant3. A comparison of the respective charging spectra reveals that most charging in that study resulted from direct primary stopping in the test mass.

Several potential charging mechanisms were assessed independently of the simulation. Some x-ray processes were found to be able to contribute to TM charging at a maximum level of $\pm 1+\mathrm{e} / \mathrm{s}$. More significantly, the kinetic emission of low energy electrons due to bombardment of the sensor by electrons and ions was identified as a more serious concern. The net charging from kinetic emission will depend on a number of factors, but even a perfect cancellation of the emission across the IS gaps will not avoid some acceleration noise. Although there are considerable uncertainties in these estimates, it is clear that 
Table 4

Summary of Test-Mass Charging in LISA

\begin{tabular}{|c|c|c|c|c|c|c|}
\hline primary & solar & chargin & rate & charging fluctu & tions & $R_{e f f}$ \\
\hline particle & activity & $R \pm \sigma_{M},+\mathrm{e} / \mathrm{s}$ & $\delta+\gamma,+\mathrm{e} / \mathrm{s} \dagger$ & $S_{R}, \mathrm{e} / \mathrm{s} / \mathrm{Hz}^{1 / 2}$ & $S_{T} \ddagger$ & $\mathrm{e} / \mathrm{s}$ \\
\hline $\mathrm{p}$ & & $41.2 \pm 0.8$ & 21 & 21.4 & - & - \\
\hline${ }^{4} \mathrm{He}$ & $\min$ & $7.7 \pm 0.3$ & 9 & 10.7 & - & - \\
\hline${ }^{3} \mathrm{He}$ & & $1.04 \pm 0.05$ & - & 4.0 & - & - \\
\hline total & & $49.9 \pm 0.9$ & 30 & 24.2 & 28.4 & 403 \\
\hline $\mathrm{p}$ & & $19.7 \pm 0.6$ & 13 & 17.4 & - & - \\
\hline${ }^{4} \mathrm{He}$ & $\max$ & $3.5 \pm 0.2$ & 5 & 8.9 & - & - \\
\hline${ }^{3} \mathrm{He}$ & & $0.44 \pm 0.03$ & - & 2.8 & - & - \\
\hline total & & $23.7 \pm 0.6$ & 18 & 19.7 & 22.5 & 254 \\
\hline
\end{tabular}

$\dagger$ Average kinetic emission as given in Table 3.

$\ddagger$ Including $\delta+\gamma$ and continuous discharge at a rate $-R$.

this effect can rival the $\mathrm{MC}$ rates, and even eclipse the contributions of GCR nuclei beyond $\mathrm{H}$. In this context we believe that the experimental determination of IIEE yields at relativistic GCR energies merits future attention.

Shot-noise fluctuations with a spectral density of $24+\mathrm{e} / \mathrm{s} / \mathrm{Hz}^{1 / 2}$ are predicted at solar minimum (i.e. charge fluctuations of $\left.24 /(2 \pi f)+\mathrm{e} / \mathrm{Hz}^{1 / 2}\right)$. That figure increases to $28.4+\mathrm{e} / \mathrm{s} / \mathrm{Hz}^{1 / 2}$ if one includes the effect of kinetic emission and considers a continuous charge neutralisation exactly balanced at a rate $-R$. A charging rate of $400 \mathrm{H} / \mathrm{s}$ in single charges would be required to produce this level of fluctuations. The acceleration noise caused by charging could reach $4 \times 10^{-16} \mathrm{~m} / \mathrm{s}^{2} / \mathrm{Hz}^{1 / 2}$ at $f=0.1 \mathrm{mHz}$ for typical IS parameters [8], making charging disturbances one of the dominant noise sources at low frequencies.

Charging from SEP events was also investigated. Some 10 events per year are expected to affect the LISA science data at solar maximum, which is not a critical scenario. Further work is under way to consolidate these predictions and to qualify the need for independent in-flight diagnostic tools such as particle monitors.

\section{Acknowledgements}

This work was supported by the European Space Agency through the SEPTIMESS project under Contract No. 16339/02/NL/FM. The authors wish to thank the CERN IT Division for facilitating access to the LSF Services. 
Thanks are also due to the Geant4 Collaboration for their effort in responding to our requirements. We thank S. Merkowitz (NASA/GSFC) for providing the LISA solid model, P. Sarra (CGS) for the LTP sensor model, N. Dunbar (EADS Astrium UK) and H. Stockburger (EADS Astrium Germany) for the SMART-2 spacecraft model, and D. Smart (SSTD/RAL) for the caging mechanism design. Thanks are due to C. Grimani (U. Urbino/INFN), H. Vocca (U.Perugia/INFN), E. Daly (TOS-EES/ESTEC), F. Lei (QinetiQ), G.Santin (TEC-EES/ESTEC) and L.Desorgher (U.Bern) for their assistance and suggestions.

\section{References}

[1] K.Danzmann et al., LISA Pre-Phase A Report (2nd Ed.) MPQ 233 (1998).

[2] S.Vitale et al., Nucl. Phys. B 110 (1998) 209.

[3] Y.Jafry, T.J.Sumner, S.Buchman, Class. Quantum Grav. 13 (1996) A97.

[4] Y.Jafry, T.J.Sumner, Class. Quantum Grav. 14 (1997) 1567.

[5] T.J.Sumner, Y.Jafry, Adv. Space Res. 25(6) (2000) 1219.

[6] H.M.Araújo et al., Class. Quantum Grav. 20 (2003) S311.

[7] D.N.A.Shaul et al., Class. Quantum Grav. 21 (2004) S647.

[8] D.N.A.Shaul et al., to appear in Class. Quantum Grav. (2004).

[9] GEANT - Detector Description and Simulation Tool, CERN Program Library, Long Writeup W5013, 1993.

[10] Geant4 Collaboration, Nucl. Instrum. Meth. A 506 (2003) 250; (release 06-00 was used in this work).

[11] A. Fassò et al., In: Workshop on Simulating Accelerator Radiation Environments, Santa Fe, USA (1993).

[12] H.Vocca et al., Class. Quantum Grav. 21(5) (2004) S665.

[13] P.Wass et al., in preparation (2004).

[14] LISA Integrated Solid Model (NASA/GSFC) Rev 1, Sep 2003.

[15] LISA Inertial Sensor Design Report (Carlo Gavazzi Space) LTP-RP-CGS-001.

[16] Geant4 Physics Reference Manual (http://geant4.web.cern.ch/geant4)

[17] C.Grimani et al., Class. Quantum Grav. 21(5) (2004) S629.

[18] C.Grimani, private communication.

[19] Data produced at LSF Batch Services provided by CERN IT Division. 
[20] The Geostationary Operational Environmental Satellite Program (GOES) is a joint effort of NASA and the US National Oceanic and Atmospheric Administration (NOAA) (http://spidr.ngdc.noaa.gov/spidr/).

[21] C.S.Dyer et al., IEEE Trans. Nucl. Sci. 50(6) (2003) 2038.

[22] R.A.Nymmik, Radiat. Meas. 30 (1999) 287.

[23] G.Höhler (Ed.), Particle-Induced Electron Emission I, (Springer, Vol. 122, 1991)

[24] G.Höhler (Ed.), Particle-Induced Electron Emission II, (Springer, Vol. 123, 1992)

[25] Int. Commission on Radiation Units and Measurements (ICRU) Rep. 55, 1995.

[26] L.Reimer, H.Drescher, J. Phys. D 10 (1977) 805.

[27] O.Benka et al., Phys. Rev. A 58(4) (1998) 2978.

[28] J.Schou, Phys. Rev. B 22(5) (1980) 2141.

[29] A.Dubus et al., Phys. Rev. B 36(10) (1987) 5110.

[30] O.Benka et al., Nucl. Instrum. Meth. B 117 (1996) 350.

[31] Stopping Power and Ranges for Electrons (ESTAR), Protons (PSTAR) and Alphas (ASTAR) (http://physics.nist.gov/PhysRefData/Star/Text).

[32] D.Liljequist, A. Appl. Phys. 16 (1983) 1567.

[33] O.Benka et al., Phys. Rev. A 52(5) (1995) 3959.

[34] J.E.Borovsky et al., Nucl. Instrum. Meth. B 30 (1988) 191.

[35] J.F.Ziegler, SRIM2003: The Stopping and Range of Ions in Matter, http://www.srim.org/\#SRIM.

[36] A.Oliva-Florio et al., Phys. Rev. B 35(5) (1987) 2198.

[37] G.I.Akap'ev et al., Tech. Phys. 43(1) (1998) 120.

[38] R.H.Day et al., J. Appl. Phys. 52(11) (1981) 6965.

[39] B.L.Henke et al., J. Appl. Phys. 52(3) (1981) 1509.

[40] W.F.Krolikowski and W.E.Spicer, Phys. Rev. B 1(2), 487.

[41] S.A.E.Johansson and J.L.Campbell, PIXE: A Novel Technique for Elemental Analysis, (Wiley, 1988).

[42] S.A.E.Johansson and T.B.Johansson, Nucl. Instrum. Meth. 137 (1976) 473.

[43] O.N.Jarvis et al., Phys. Rev. A 5(3) (1972) 198.

[44] S.M.Shafroth et al., Phys. Rev. A 7(2) (1973) 556.

[45] J.D.Jackson, Classical Electrodynamics, 3rd Ed., (Wiley, 1998). 
[46] V.M.Grichine, Nucl. Instrum. Meth. A 502 (2003) 133.

[47] V.A.Bazylev and N.K.Zhevago, Sov. Phys. Usp. 25 (1982) 565.

[48] K.Krane, Introductory Nuclear Physics, (Wiley, 1988) 465.

[49] R.Michel et al., Nucl. Instrum. Meth. B 129 (1997) 153. 